INDO GLOBAL JOURNAL OF

PHARMACEUTICAL SCIENCES

ISSN 2249- 1023

\title{
Microemulsion System: A Recent Trend in Food Packaging Industries
}

\author{
Harsha Nagar, Rashi Prakash, Arushi Pant, Kanupriya Jha, Rachana, Manisha Singh * \\ Department of Biotechnology, Jaypee Institute of Information Technology, A-10, Sector-62, Noida, Uttar Pradesh, India-201307
}

Address for Correspondance: Manisha Singh, manishasingh1295@gmail.com

\section{Keywords}

Phytoconstituents;

Flavonoid; Nitric

Oxide; ABTS

Assay; Radical

Scavenging

Activity.

\begin{abstract}
Microemulsions (ME) are dispersion of oil in water $(\mathrm{o} / \mathrm{w})$ or water in oil (w/o) with added surfactant and cosurfactant which are thermodynamically stable and have unique features of enriched reaction efficiency, extraction techniques and stabilisation. Use of microemulsions has been significantly emerged in the area of food packaging industries for its potential benefits such as improved bioavailability, antimicrobial effects, enhanced sensory acceptance and targeted delivery of bioactive compounds. It further, is a most suitable option available now a days for food packaging as it forms a protective coating to minimize weight loss and internal oxidation. Moreover, it also provides protection to food articles by minimising its enzymatic degradation, it basically act as an antimicrobial agents which help in retarding the growth and proliferation of pathogenic or spoilage microbes on food materials. Microemulsion system, itself has been reported to show good antimicrobial and antioxidant properties and hence due to this benefit of ME, it play a crucial role in food packaging industry. Also, the synergistic beneficial effect has been greatly provided between lipophilic hydrophilic antioxidant surfactants which are used under the GRAS limits. The composition of microemulsion includes various excipients such as - short chain alkols such as ethanol,polypols ( propylene glycol and glycerol), salts (organic such sodium benzoate and inorganic such as $\mathrm{NaCl}$ ) and several non-ionic surfactants such as ethoxylated sorbitan esters or sucrose esters. (C) 2016 iGlobal Research and Publishing Foundation. All rights reserved.
\end{abstract}

Conference Proceedings: International Conference on Advances in Plant and Microbial Biotechnology (PMB-2017); JIIT, Noida: February 02-04, 2017

Indo Global Journal of Pharmaceutical Sciences( ISSN 22491023 ; CODEN- IGJPAI; NLM ID: 101610675) indexed and abstracted in EMBASE(Elsevier), SCIRUS(Elsevier),CABI, CAB Abstracts, Chemical Abstract Services(CAS), American Chemical Society(ACS), Index Copernicus, EBSCO, DOAJ, Google Scholar and many more. For further details, visit http://iglobaljournal.com 